\title{
Targeting the mTOR pathway in idiopathic multicentric Castleman disease
}

\author{
Robert M. Stern ${ }^{1,2}$ and Nancy Berliner ${ }^{1,2}$ \\ ${ }^{1}$ Hematology Division, Brigham and Women's Hospital, Boston, Massachusetts, USA. ${ }^{2}$ Harvard Medical School, Boston, Massachusetts, USA
}

\begin{abstract}
Idiopathic multicentric Castleman disease (iMCD) is a rare hematologic illness of systemic inflammation and organ dysfunction, with unknown etiology. Although therapies targeting IL- 6 have been proven effective, a subset of patients with $\mathrm{M} C D$ are resistant to this approach. In this issue of the $J C I$, Fajgenbaum et al. performed an in-depth analysis of serum inflammatory markers in three IMCD patients refractory to IL-6 blockade, and identified activation of the mTOR pathway associated with symptom flares. Treatment with sirolimus, an mTOR inhibitor, induced remission in all three patients. This study models a precision medicine approach to discovering therapies for rare diseases.
\end{abstract}

\section{Idiopathic multicentric} Castleman disease and IL-6 inhibition

Idiopathic multicentric Castleman disease (iMCD) is a rare, systemic hematologic illness that involves widespread cytokineinduced inflammation, polyclonal lymphoproliferation, cytopenias, and multi-organ dysfunction. It has an incidence of 10001500 cases in the United States and a 5-year overall survival of only $55 \%-77 \%(1,2)$. Diagnostic criteria for iMCD include lymph node enlargement in two or more lymph node stations, characteristic lymph node pathology, and representative clinical and laboratory findings (3). The most severe cases of iMCD are marked by thrombocytopenia, anasarca, fever, reticulin fibrosis, and organomegaly, collectively categorized as the TAFRO subtype (4). Notably, iMCD is distinct from other forms of Castleman disease, which include unicentric Castleman disease, involving only one anatomic site and generally curable by surgical excision, and human herpesvirus 8-induced multicentric Castleman disease, which is virally mediated and arises in HIV-positive and other immunosuppressed patients.
The etiologic trigger for $\mathrm{MMCD}$ remains unknown. However, excessive signaling from the proinflammatory cytokine IL-6 appears to play a role in the pathogenesis and symptomatology of the disease (Figure 1A). IL-6 is a multifunctional cytokine involved in inflammation, immune response, and hematopoiesis. It is produced by numerous cell types including monocytes, macrophages, lymphocytes, fibroblasts, and endothelial cells. Among its functions, IL-6 induces plasmacytosis, hypergammaglobulinemia, thrombocytosis, inflammatory protein production, and activation of macrophages and $\mathrm{T}$ cells, all of which can be present in iMCD (5). IL-6 may also induce expansion of autoantibody-producing, CD5-positive lymphocytes, leading to the autoimmune phenomena of $\operatorname{iMCD}(6,7)$. The connection between IL- 6 and iMCD has been demonstrated in both humans and animal models. Mice infected with an IL-6-expressing recombinant retrovirus develop an iMCD-like syndrome, which resolves after administration of an anti-IL-6 mAb $(8,9)$. Similarly, humans who are given pharmacologic doses of recombinant IL-6 or who

Related Article: p. 4451

Conflict of interest: The authors have declared that no conflict of interest exists.

have an IL-6-secreting malignancy develop iMCD-like syndromes $(10,11)$.

Identification of the role of excessive IL-6 signaling in the pathogenesis of iMCD led to the development of novel iMCD therapies targeting IL-6. Prior to these treatments, iMCD therapy was nonstandardized and often included a combination of steroids, rituximab, and/or multi-agent chemotherapy. While these regimens had a high initial response rate, relapse was common and side effects were significant (2). The development of siltuximab, an IL-6-neutralizing mAb, and tocilizumab, a humanized $\mathrm{mAB}$ that binds to and blocks the IL- 6 receptor, changed the treatment paradigm. In a randomized, double-blind, placebo-controlled phase 2 trial, approximately one-third of patients treated with siltuximab were found to have a durable complete or partial response, whereas no patients treated with placebo responded (12). Similarly, in a nonrandomized prospective study of tocilizumab in iMCD, approximately half of the patients had a significant reduction in their lymphadenopathy and improvement in their iMCD symptoms (13). Based on these results, siltuximab was approved for treatment of iMCD in the United States, Canada, and Europe, and tocilizumab was approved for treatment of iMCD in Japan. In addition, IL-6 blockade is now considered first-line therapy for the treatment of all patients with iMCD (2).

\section{PI3K/Akt/mTOR pathway upregulation in IL-6 blockade- refractory iMCD}

While IL-6 blockade represented a significant breakthrough in iMCD treatment, a substantial portion of patients with iMCD remain refractory to therapy with siltuximab or tocilizumab. This suggests that additional pathways may underlie iMCD pathogenesis and be important targets for future iMCD therapies. Determination of these additional pathways, however, is limited by an absence of cell lines, animal 


\section{IL-6 signaling pathway}

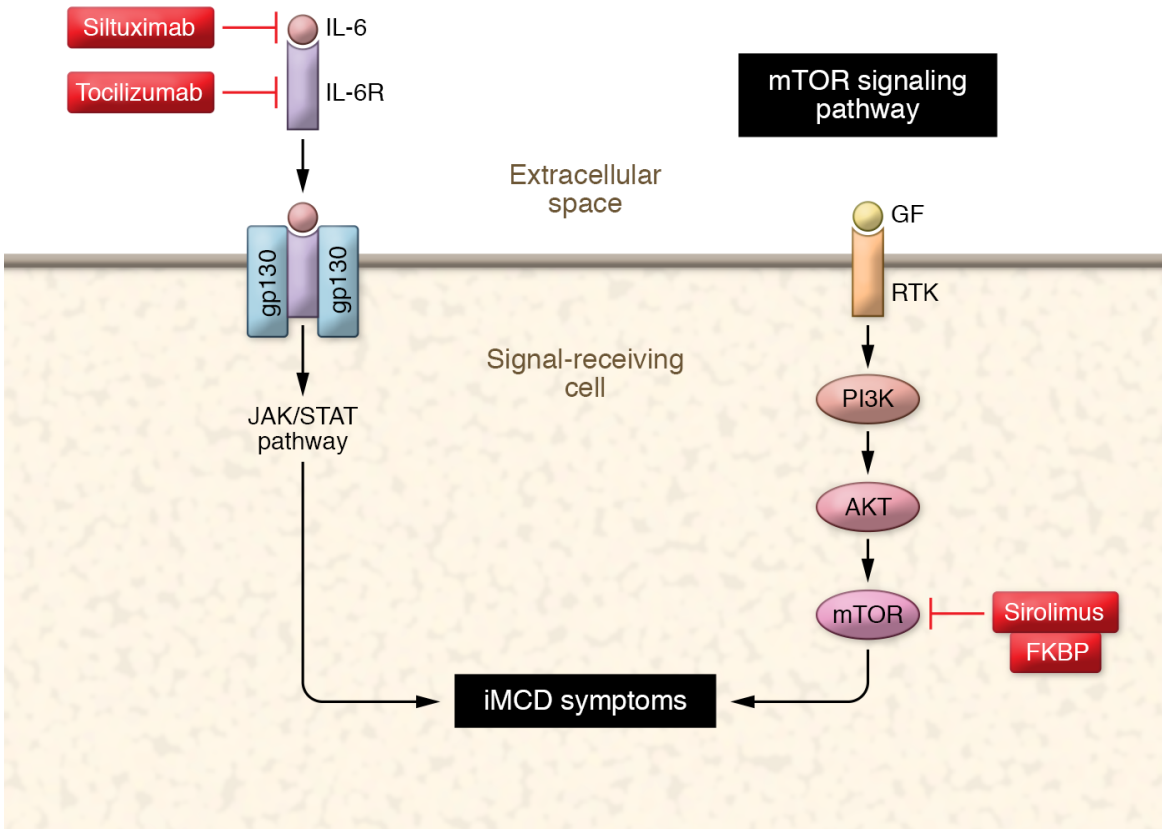

Figure 1. Inhibition of the IL-6 and mTOR pathways in IMCD influences symptoms. IL- 6 and IL-6 receptor (IL-6R) associate with the signal transducer gp130, leading to dimerization and activation of the JAK/STAT signaling cascade. Siltuximab neutralizes IL- 6 and tocilizumab blocks the IL-6R. Growth factor (GF) binds to the receptor tyrosine kinase (RTK) leading to downstream activation of PI3 kinase (PIBK), AKT, and ultimately mTOR. Sirolimus binds to the tacrolimus binding protein (FKBP), and together sirolimus and FKBP inhibit mTOR activity.

models, or large data sets of IL-6 blockaderefractory iMCD.

In this issue, Fajgenbaum et al. identify the mTOR pathway as a pharmacologically targetable pathway within IL-6 blockaderefractory iMCD (Figure 1B) (14). mTOR is an atypical serine/threonine protein kinase that is part of the phosphoinositide 3-kinase-related (PI3K-related) kinase family (15). Upon activation, mTOR interacts with several downstream proteins, ultimately leading to the formation of two distinct complexes: mTORC1 and mTORC2. The mTOR signaling pathway is responsible for both sensing and integrating environmental cues that regulate growth and homeostasis, and is known to be altered in many diseases including cancer, obesity, type 2 diabetes, lymphangioleiomyomatosis, and neurodegenerative diseases (15).

Fajgenbaum et al. pinpointed the $\mathrm{mTOR}$ pathway using a novel precision medicine approach that utilized in-depth analyses of blood and tissue samples from three IL-6 blockade-refractory patients with the TAFRO subtype of iMCD (14). The authors first measured serum inflammatory mark- ers in one patient both before and during an iMCD flare and found that the soluble IL-2 receptor alpha chain (sIL-2R $\alpha)$, a marker of $\mathrm{T}$ cell activation, and VEGF-A were the only two serum inflammatory markers that had a consistent upward trend. Next, the authors employed serum-based proteomics to assess differential analyte expression during flare and remission in this same patient, and once again discovered that sIL-2R $\alpha$ and VEGF-A were two of the most elevated serum proteins at the onset of a flare. Finally, the authors utilized three separate platforms to identify upregulated pathways in this patient at the time of flare. Each platform identified the PI3K/Akt/ mTOR pathway as enriched during a flare and inclusive of both sIL-2R $\alpha$ and VEGF-A.

Having recognized the mTOR pathway as a potential target for one patient, the authors then sought to confirm this finding in two additional patients and through additional laboratory techniques. To start, the authors performed flow cytometry on peripheral blood mononuclear cells from all three patients during relapse and found that, compared with age-matched healthy controls, activated $\mathrm{CD}^{+} \mathrm{T}$ cells were increased in patients during flare. They also noted an increase in $\mathrm{CD}^{+} \mathrm{T}$ cells at the level of the patient's lymph nodes. Next, the authors measured VEGF-A levels in the two additional patients during flares and found that the levels were well above the normal range. Finally, they discovered that ribosomal protein $\mathrm{S} 6$ phosphorylation, a read-out of mTOR activity, was both extensively and specifically elevated in the interfollicular lymph node space of all three patients.

\section{Treatment with sirolimus in IL-6 blockade-refractory iMCD}

Based on the above findings, as well as the limited remaining therapeutic options for IL-6 blockade-refractory patients, Fajgenbaum et al. made the jump to treat each patient with sirolimus. Sirolimus is a known antiproliferative and immunosuppressive drug that directly inhibits mTOR (16). It is generally well-tolerated and is used in several conditions including after heart and kidney transplantation, lymphangioleiomyomatosis, and autoimmune lymphoproliferative syndrome. Sirolimus treatment normalized VEGF-A levels and decreased circulating activated $\mathrm{CD} 8^{+}$ $\mathrm{T}$ cells in all three patients. Moreover, the patients have tolerated the sirolimus without significant side effects, have experienced symptomatic benefit, and have been in remission for 64,17 , and 17 months, respectively (14).

\section{Conclusions and remaining questions}

Fajgenbaum et al. successfully employ a precision medicine approach to identify and target the mTOR pathway in 3 IL-6 blockade-refractory patients with iMCD (14). Their methodology was innovative and presents a potential roadmap for similar investigations in other rare diseases. In addition, their findings serve as an excellent starting point for future trials that will investigate both the efficacy and safety of sirolimus in the treatment of IL- 6 blockade-refractory iMCD patients. Nevertheless, several unanswered questions remain. First, while Fajgenbaum et al. convincingly demonstrate the role of mTOR signaling in IL-6 blockade-refractory iMCD, the etiologic trigger of iMCD and whether mTOR 
signaling is associated with this trigger remain unknown. Second, it is not evident whether the authors' findings will be generalizable across a larger cohort of IL- 6 blockade-refractory iMCD patients. This is of particular concern since the TAFRO subtype of iMCD has been shown to have different proteomic profiles from other subtypes of iMCD $(17,18)$. Third, there are multiple pharmacologic agents that target the mTOR pathway, and it remains to be determined whether sirolimus is the optimal choice. Finally, many cytokines are expressed in locally restricted sites, limiting the capacity of serum proteomics to detect their role in disease pathogenesis, even when their impact is systemic. Therefore, studies of other rare diseases will be needed to demonstrate whether the authors' methodology can be adapted to a wider spectrum of inflammatory syndromes.

Address correspondence to: Nancy Berliner, Brigham and Women's Hospital, 75 Francis Street, Boston, Massachusetts 02115, USA. Phone: 617.732.5840; Email: nberliner@bwh.harvard.edu.
1. Simpson D. Epidemiology of Castleman disease. Hematol Oncol Clin North Am. 2018;32(1):1-10.

2. van Rhee F, et al. International, evidence-based consensus treatment guidelines for idiopathic multicentric Castleman disease. Blood. 2018;132(20):2115-2124.

3. Fajgenbaum DC, et al. International, evidencebased consensus diagnostic criteria for HHV-8negative/idiopathic multicentric Castleman disease. Blood. 2017;129(12):1646-1657.

4. Liu AY, et al. Idiopathic multicentric Castleman's disease: a systematic literature review. Lancet Haematol. 2016;3(4):e163-e175.

5. Kishimoto T. IL-6: from its discovery to clinical applications. Int Immunol. 2010;22(5):347-352.

6. Hall PA, Donaghy M, Cotter FE, Stansfeld AG, Levison DA. An immunohistological and genotypic study of the plasma cell form of Castleman's disease. Histopathology. 1989;14(4):333-46; discussion 429.

7. Menke DM, et al. Diagnosis of Castleman's disease by identification of an immunophenotypically aberrant population of mantle zone B lymphocytes in paraffin-embedded lymph node biopsies. Am J Clin Pathol. 1996;105(3):268-276.

8. Brandt SJ, Bodine DM, Dunbar CE, Nienhuis AW. Dysregulated interleukin 6 expression produces a syndrome resembling Castleman's disease in mice. J Clin Invest. 1990;86(2):592-599.

9. Katsume A, et al. Anti-interleukin 6 (IL-6) receptor antibody suppresses Castleman's disease like symptoms emerged in IL-6 trans- genic mice. Cytokine. 2002;20(6):304-311.

10. Momoi A, et al. IL-6-positive classical Hodgkin's lymphoma co-occurring with plasma cell type of Castleman's disease: report of a case. Int J Hematol. 2013;97(2):275-279.

11. van Gameren MM, et al. Effects of recombinant human interleukin- 6 in cancer patients: a phase I-II study. Blood. 1994;84(5):1434-1441.

12. van Rhee F, et al. Siltuximab for multicentric Castleman's disease: a randomised, doubleblind, placebo-controlled trial. Lancet Oncol. 2014;15(9):966-974.

13. Nishimoto N, et al. Humanized antiinterleukin-6 receptor antibody treatment of multicentric Castleman disease. Blood. 2005;106(8):2627-2632.

14. Fajgenbaum DC, et al. Identifying and targeting pathogenic $\mathrm{PI} 3 \mathrm{~K} / \mathrm{AKT} / \mathrm{mTOR}$ signaling in IL- 6 blockade-refractory idiopathic multicentric Castleman disease. J Clin Invest. 2019;129(10):4451-4463.

15. Laplante M, Sabatini DM. mTOR signaling in growth control and disease. Cell. 2012;149(2):274-293.

16. Kahan BD. Sirolimus: a comprehensive review. Expert Opin Pharmacother. 2001;2(11):1903-1917.

17. Iwaki $\mathrm{N}$, et al. Elevated serum interferon $\gamma$-induced protein $10 \mathrm{kDa}$ is associated with TAFRO syndrome. Sci Rep. 2017;7:42316.

18. Pierson SK, et al. Plasma proteomics identifies a 'chemokine storm' in idiopathic multicentric Castleman disease. Am JHematol. 2018;93(7):902-912. 